\title{
Sonographic Evaluation of Benign Pelvic Masses
}

\author{
Aleksandar Ljubic, Tatjana Bozanovic, Zoran Vilendecic \\ Institute for Obstetrics and Gynecology, Clinical Center of Serbia, Belgrade, Serbia
}

\section{INTRODUCTION}

Although the sonographic features of a pelvic mass frequently do not permit a specific histopathologic diagnosis, sonography usually provides clinically important parameters for the evaluation of a pelvic mass. Pelvic sonography can confirm the presence or absence of a suspected pelvic mass. Sonographic features, such as size, consistency, shape, probable origin and relationship of the mass to other pelvic structures can be valuable parameters in a decision-making process. ${ }^{1}$

A pelvic mass may be gynecologic in origin or it may arise from the urinary tract or bowel. With the respect to gynecologic causes, lesion can be uterine or adnexal, predominantly ovarian.

\section{ADNEXAL MASSES}

Pelvic masses derived from adnexal structures can appear as cystic lesions and less frequently as solid masses. In general, functional ovarian cysts are the most common single, welldefined adnexal masses in women of reproductive age. Other cystic masses include hydrosalpinges, parovarian cysts, and endometriomas.

\section{Functional Ovarian Cysts}

During the reproductive years the majority of adnexal masses are follicle cysts. These tumors are functional in nature and result from abnormalities that occur at different stages of folliculogenesis. These include follicular cysts, corpus luteum cysts, and theca lutein cysts. In general, they vary in size, ranging from a few to $8-10 \mathrm{~cm}$ in diameter, rarely cause symptoms and disappear in 1 to 3 months.

The most common follicular cysts occur due to failure of a mature follicle to rupture at the time of ovulation. Such cysts are larger than $3 \mathrm{~cm}$ and usually asymptomatic. They may regress spontaneously or after a clinical trial of hormonal suppression. Larger lesions may cause heaviness in the lower pelvis or in the leg, and in the case of rupture; they can cause pain and peritoneal signs. Anechoic, thin-walled and well-defined change which does not disturb the contour of the ovary can be documented on sonography (Fig. 1).

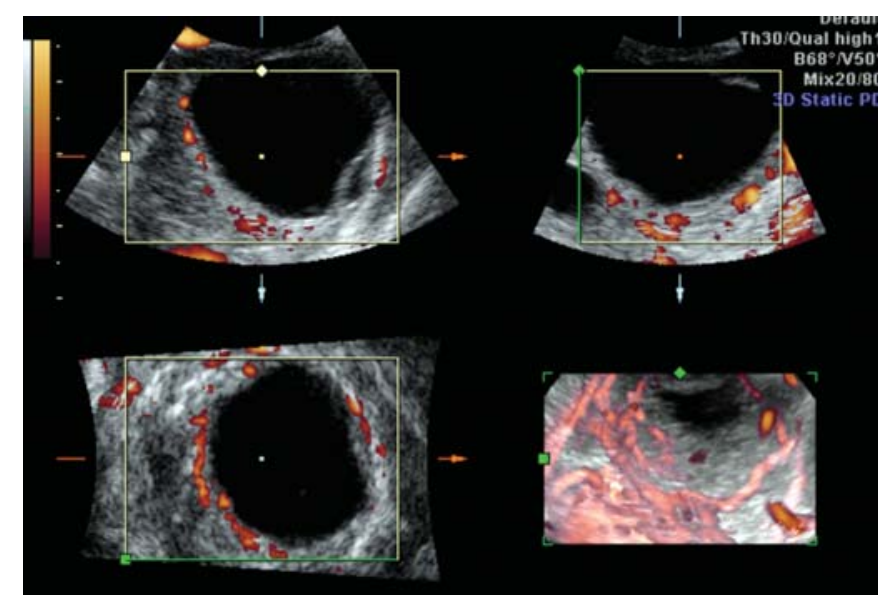

Fig. 1: Functional ovarian cyst. 3D power color Doppler

Corpus luteum cysts form from continued hemorrhage and collection of blood within the corpus luteum. They are rarely larger than $5 \mathrm{~cm}$ in diameter, and usually have a thicker wall than follicular cysts. Generally, they regress within a few weeks. If rupture leading to hemoperitoneum occurs, surgical intervention is required. Sonographic features can be variable, depending on the amount and organization of internal clot. The most common appearance of a corpus luteum cyst is a complex mass with internal echoes but with enhanced transmission. While fibrinolyzed clot is typically hypoechoic, acute intraparenchymal hemorrhage results in an irregular echogenic area. The cyst wall may be irregular in contour due to a clot that is adherent to it. In addition, corpus luteum cyst can mimic solid ovarian mass, such as teratoma.

Theca lutein cysts are the least common functional ovarian cysts resulting from excessive human chorionic gonadotropin stimulation. They are usually bilateral and occur in association with molar pregnancies, multiple gestations, ovulation induction and $\mathrm{GnRH}$ analogs use. A large (up to $30 \mathrm{~cm}$ ), multicystic and thin-walled anechoic change is visualized. Sonographic evaluation of the uterus should be documented. They regress spontaneously.

Ovarian hyperstimulation syndrome is a life-threatening complication of ovulation induction. The disorder ranges from 
mild form with abdominal discomfort, probably due to distention of ovarian capsule to severe life-threatening form with hemoconcentration, oliguria, and elevated serum creatine. Pleural effusions and ascites can be present. Ovarian hyperstimulationlike conditions may occur in women suffering from trophoblastic disease. Hyperstimulation tends to occur when there are several small or intermediate-sized follicles, although this syndrome can be more accurately predicted by extremely high levels of estradiol (over $3000 \mathrm{pg} / \mathrm{mL}$ ). Sonographic features include bilaterally enlarged ovaries with hypoechoic areas which correspond to follicles. The peritoneal fluid in cul-de-sac may be noted (Fig. 2).

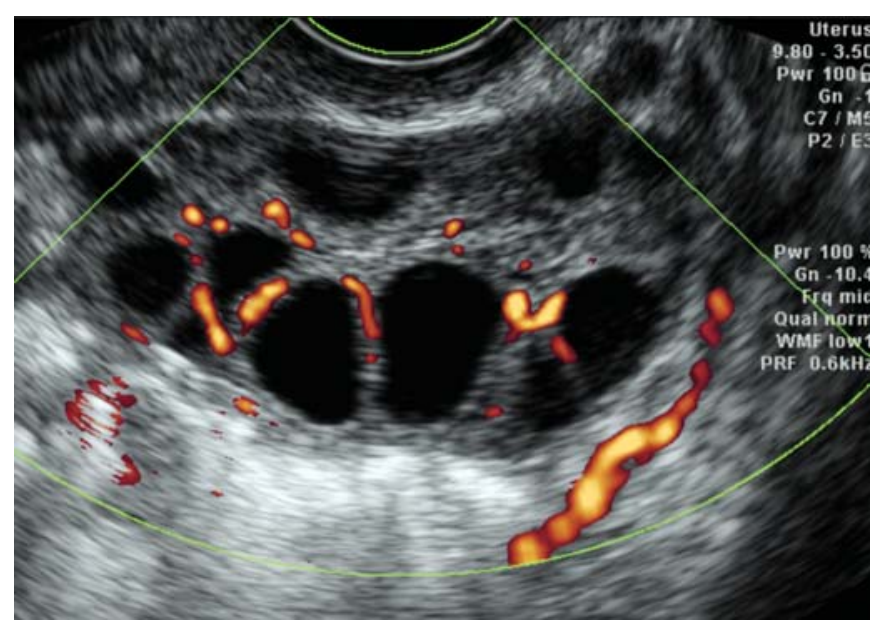

Fig. 2: Ovarian hyperstimulation

\section{Endometrioma}

Endometriosis is characterized by the presence and growth of the glands and stroma identical to the lining of the uterus in an ectopic location. Endometriomas are small areas of endometriosis that can be identified macroscopically. Local inflammatory response results in fibrosis and thickening of the adnexal ligaments. Adhesions of adjacent pelvic structures may be formed. There is a spectrum of sonographic findings in endometriosis, depending on the size and number of endometriomas present and their internal contents. Typical endometriotic implants on the uterosacral ligaments, peritoneum or surface of the bowel are difficult to depict with sonography. Sporadically, they present as echogenic areas within the culde-sac. Ovarian endometriomas, termed also as chocolate cysts, may enlarge to 6-8 cm in size. Nonspecific, single or multiple cystic ovarian changes with low-level echoes can be documented (Figs 3 and 4). Due to similar sonographic appearance, these ovarian endometriomas sometimes can not be confidently distinguished from corpus luteum cysts. Large

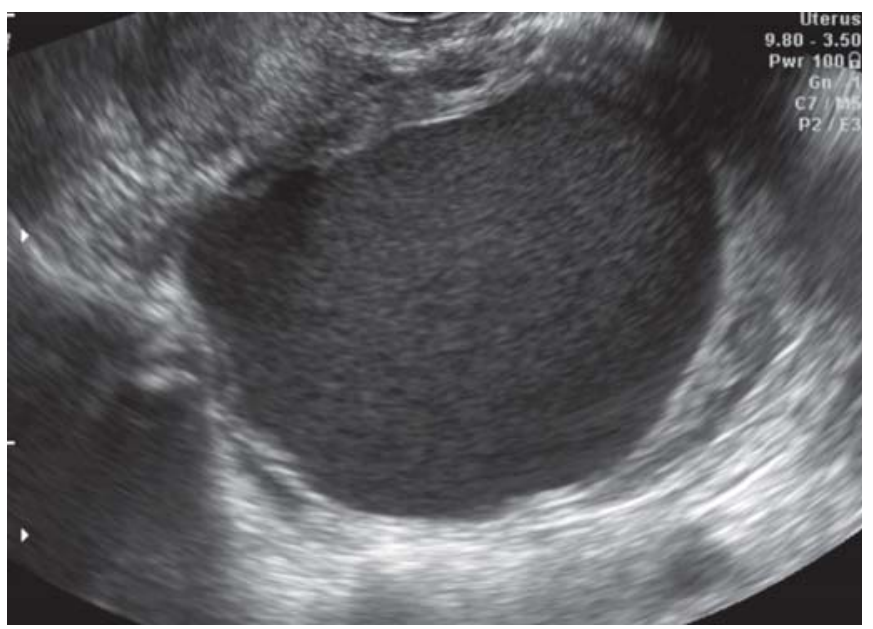

Fig. 3: Endometriosis

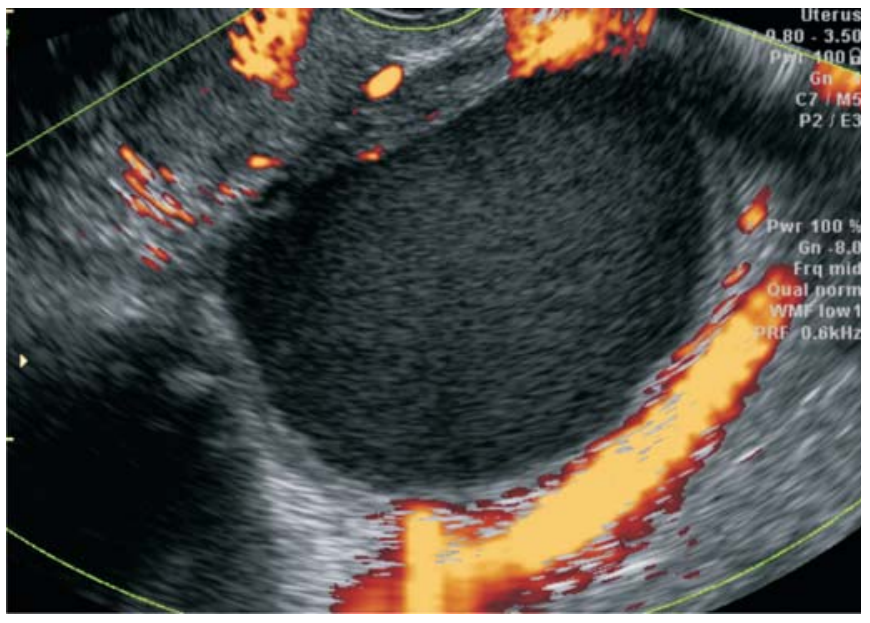

Fig. 4: Endometriosis. Color power Doppler

ovarian endometrioma may rupture into peritoneal cavity. In such rare instances, surgical intervention is warranted. Pelvic ultrasound may give additional and confirmatory information but can not be used for primary screening. Nevertheless, sonography is a useful means to monitor the effectiveness of medical therapy of the more extensive cases.

\section{Benign Ovarian Neoplasms}

Cystadenomas are the most common type of cystic ovarian tumors. These tumors arise from surface epithelium that covers the ovary. They are most frequently encountered in postmenopausal women. In the early stages of development, cystadenomas may have a similar appearance to other cystic adnexal masses. When they enlarge, they frequently contain characteristic internal components, such as septations or papillary excrescences, which allow their sonographic 
recognition. The surface epithelial cells can secrete either a mucinous or a serous substance. Serous cystadenomas are cystic ovarian masses and are bilateral in $20 \%$. They are often multilocular. Sonographic features include a smooth wall, sometimes with papillary components, no internal echoes and enhanced transmission. Psammoma bodies, which are areas of fine calcific granulation, may be visualized. Mucinous cystadenomas typically have a lobulated, smooth surface, often multilocular, and may be bilateral in up to $10 \%$ of cases. Mucoid material which is present within the cystic structures results in low-level echoes on sonography. Projections of solid tissue can be observed. Papillary excrescences are not present. Since these tumors are large they are easily visualized by transabdominal approach.

Although sonographic findings can vary, benign diagnosis should be assumed only for lesions that do not have solid tissue on imaging findings. In general, larger amount of solid areas or irregular internal morphology adds in favor of malignancy.

A Brenner tumor is a benign transitional cell tumor which is often found incidentally. Such tumor presents as a unilateral and well-defined, solid nodule usually with another cystic mass, typically a mucinous cystadenoma.

Dermoid cysts or benign cystic teratomas are the most common type of germ cell tumor encountered in patients of childbearing age. They are bilateral in $10 \%$ of cases. Histologically, benign cystic teratomas have a mixture of elements from all dermal layers, including fat, skin, teeth, and hair. Sonographic appearance varies, depending on the internal components of the tumor. Typically, a mixed, predominantly solid mass with hyperechogenic, calcified structures and cystic areas is observed. Nevertheless, sonographic appearances can range from completely cystic structure filled with sebaceous fluid to almost completely solid lesions consisting of soft tissue. The high-fat content of most dermoid cysts allows them to float within the abdominal and pelvic cavity. Due to localization and sonographic features, such tumors can mimic gas- or fecesfilled bowel loops. The absence of peristalsis and the presence of distal acoustical shadowing in lesions with large amount of fat, may be a helpful indicator of the presence of a dermoid cyst (Figs 5 to 7 ).

\section{Pelvic Inflammatory Disease}

Pelvic inflammatory disease refers to inflammation of the upper genital tract. Infection of the tubes is the most characteristic and common component of pelvic inflammatory disease. It is usually a polymicrobial infection caused by organisms

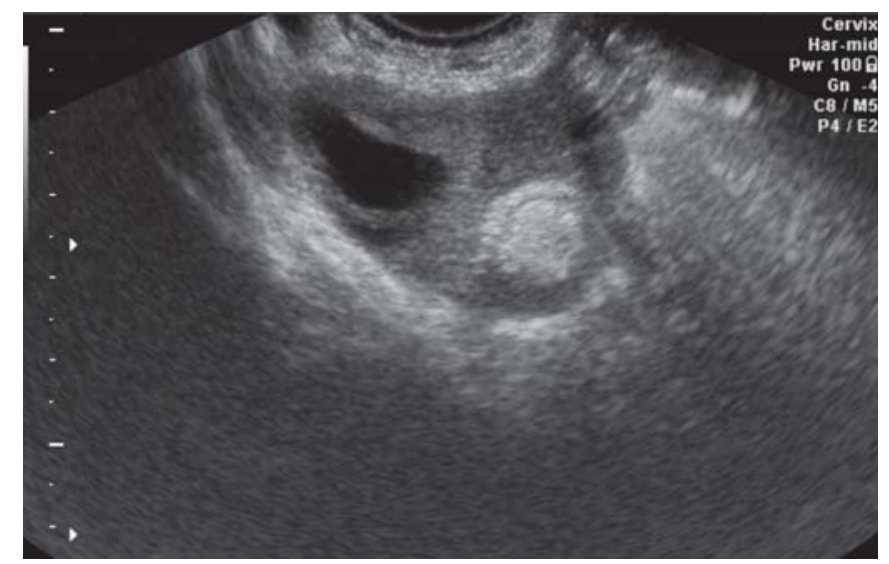

Fig. 5: Dermoid cyst

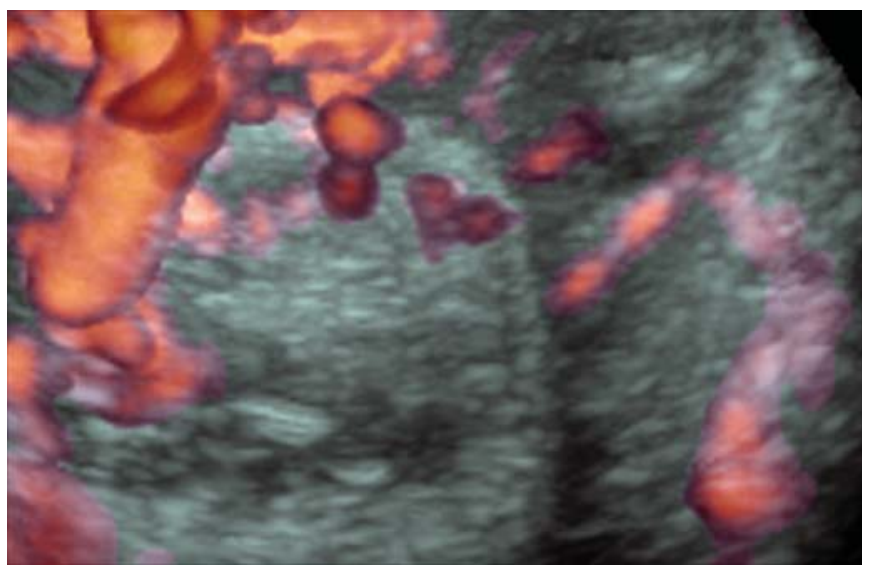

Fig. 6: Dermoid cyst. 3D power color Doppler

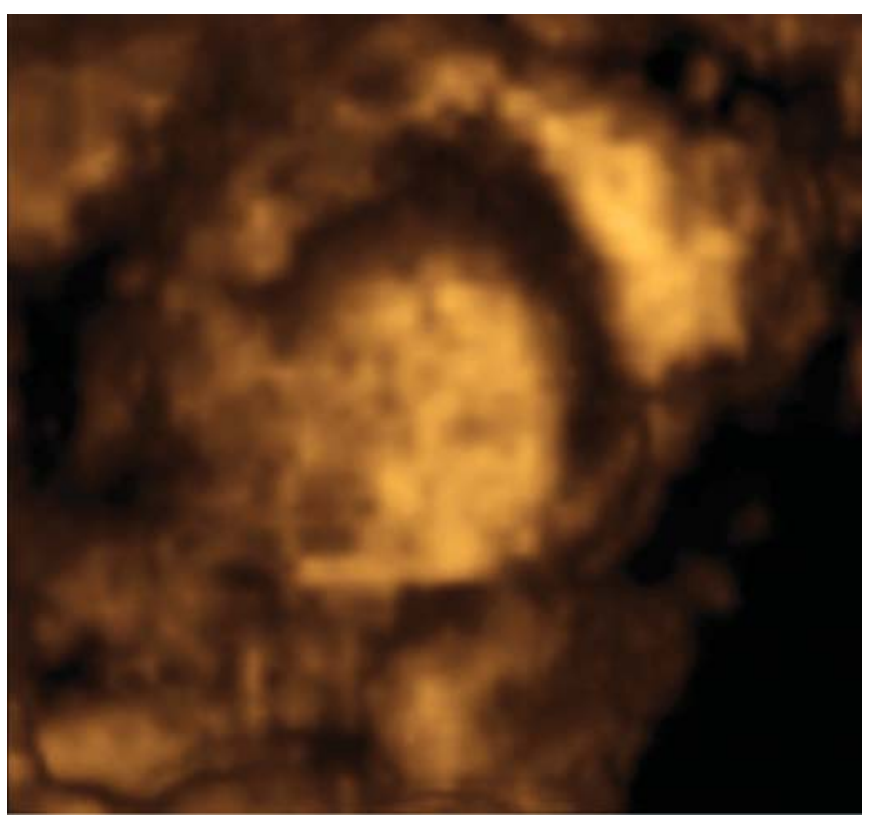

Fig. 7: Dermoid cyst (4D) 
ascending from the vagina and cervix along the mucosa of the endometrium to infect the mucosa of the oviduct. Microorganisms found in culture specimens are $N$. gonorrheae, C. trachomatis, aerobic and anaerobic bacteria in majority of cases. However, the type and number of species vary depending on the stage of the disease when the culture is obtained. Despite the lack of sensitivity, ultrasonography may be confirmatory of symptoms and physical signs. The changes are nonspecific and include dilated and fluid-filled tubes, free peritoneal fluid, uni or multilocular adnexal masses with irregular borders and thickened wall. The uterus is slightly enlarged with blurred borders and well-defined medial echo due to collection of inflammatory exudates in endometrial cavity.

As a result of tubal obstruction pyosalpinx may form. Sonography reveals tubular or fusiform anechoic change with echoic walls. If the inflammatory process involves the ovary as a part of the wall, a tubo-ovarian abscess may be produced. It has a more complex, usually multicystic appearance, with lowlevel echoes representing pus or cellular debris and echoes arising from connective tissue. In most cases, tubo-ovarian abscesses can be distinguished from other pelvic masses by verifying that the abscess cavity is within the confines of the ovary (Fig. 8).

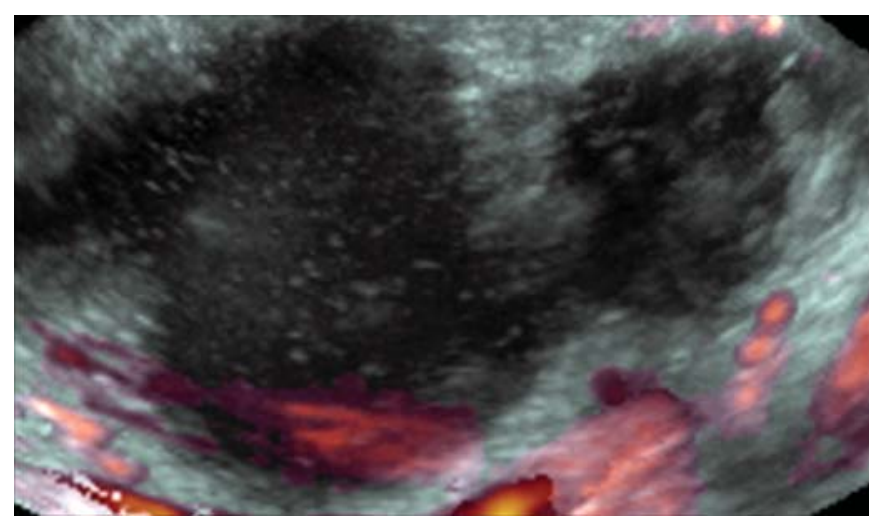

Fig. 8: Pelvic inflammatory disease. 3D color power Doppler

Hydrosalpinx is an end stage of pyosalpinx. A collection of watery, sterile fluid in the fallopian tube results in a fusiform, anechoic or hypoechoic adnexal mass of variable size. The dilated tube can be traced to the corneal area of the uterus. The visualization of ovary and the lack of peristalsis can be helpful in differentiating from ovarian cysts or small bowel loops.

Ectopic pregnancy can present as a complex and sometimes solid adnexal mass. ${ }^{2}$ The topic of ectopic pregnancy is extensively covered.

\section{Other Less Common Cystic Masses}

A variety of masses that do not arise directly from the uterus or ovaries can appear as adnexal cystic masses. The most common one is the paraovarian cyst, which arises from wolffian duct remnants in the mesovarium. This type of mass usually measures from 3 to $5 \mathrm{~cm}$ but can be as large as a pelvoabdominal cystadenoma. Occasionally, these cysts contain hemorrhage; rarely they contain internal septations. Like other adnexal cysts, these masses may potentiate torsion of the adnexa.

Another cystic mass that can be encountered in the patient with previous surgery is a peritoneal inclusion cyst. The cysts result from serous fluid collections that become entrapped by adhesions or overlaps of pelvic peritoneum related to previous pelvic surgery. Paraovarian cysts and tumors can usually be distinguished from ovarian ones by their location. As in ovarian tumors, paraovarian ones that contain solid areas or septation should be considered as potentially malignant. Cysts arising from the fimbriated end of the tube (cysts of Morgagni) may appear as cystic adnexal masses and are best seen if there is fluid surrounding the tube.

Inclusion cysts of the cervix (nabothian cysts) can usually be distinguished from other adnexal cysts in that they can be delineated to be within the confines of the uterine cervix and endocervical canal. Nabothian cysts are typically a few millimeters in size and can be distinguished from Gartner's duct cysts, which appear as tubular cystic structures in the upper vagina and (less frequently) within the uterine wall. Distended arcuate veins that course along the outer third of the myometrium can also appear as punctate cystic structures within the confines of the uterus. Calcifications within the arcuate arteries can be observed, particularly in postmenopausal women.

\section{Torsed Ovary}

An enlarged ovary has the potential for attenuating its pedicle and torsing along its axis. Masses within the ovary, such as those resulting from hemorrhagic corpus luteum, can potentiate torsion. There are no specific sonographic findings in ovarian torsion. Usually, the ovary is enlarged and contains a single hypoechoic area representing a hemorrhagic area. If the torsion is intermittent and incomplete, massive stromal edema of the ovary can result. In these cases, the ovary is diffusely enlarged and contains multiple hypoechoic and echogenic areas resulting from hemorrhagic infarcts. Intraperitoneal fluid may be present, related to obstruction of venous and lymphatic return, resulting in a transudate from the capsule of the ovary. Duplex Doppler examination of the ovarian blood supply along the ovarian and 
infundibulopelvic ligaments may facilitate the specific diagnosis of ovarian torsion by demonstrating absent or high-impedance arterial flow and absent venous flow. ${ }^{3,4}$

One relatively rare cause of a complex mass in a patient presenting with pain is an ovarian remnant. This ovarian tissue left behind after difficult oophorectomy may contain hemorrhagic corpus luteum appearing as a hypoechoic area with internal strands. These strands may obstruct the distal ureter. Peritoneal cysts, which also form after pelvic surgery, tend to envelope the ovary rather than arise from within it. Therefore, it is important to delineate the presence or absence of a parenchymal rim around the mass.

\section{SOLID PELVIC MASSES}

In general, most solid pelvic masses are of uterine, rather than of ovarian, origin. Therefore, distinction of the internal consistency of a pelvic mass is indirectly helpful in establishing a uterine or ovarian origin.

\section{Ovarian Masses}

Compared with the frequency of solid masses representing uterine leiomyomata, ovarian solid masses are less common. Solid ovarian tumors include adenocarcinoma, fibromas, and thecomas. Some rapidly growing epithelial tumors of the ovary, such as cystadenofibromas, may also appear as solid. Fibromas and thecomas may be associated with benign ascitic fluid.

Ovaries that undergo partial or intermittent torsion, or both, may appear as a solid mass. These ovaries are usually extremely edematous related to the obstruction in venous return. Fibromas typically appear as solid masses that demonstrate marked attenuation due to the fibrous tissue elements. Cystadenofibromas may appear as solid masses with areas of calcification, similar to the sonographic findings of a extrauterine fibroid. Other less common solid ovarian tumors can arise from metastasis from the gastrointestinal tract, lymphomas, and other primary neoplasms.

\section{Other Solid Masses}

Rarely, tubal disorders, such as carcinomas, produce solid adnexal masses. These are best depicted using transvaginal sonography. Some nongynecologic masses that can appear solid include lymphadenopathy, masses related to the bowel, or both. Lymphadenopathy can be recognized by its typical location in the iliac or para-aortic regions, or both. Only massively enlarged groups of lymph nodes can be detected on sonography, and they usually appear as a lobulated, hypoechoic mass in the expected region of a major lymph node chain. Solid masses resulting from bowel tumors can be diagnosed by the typical echogenic center arising from the bowel lumen. Ectopic pelvic kidneys may be located superior to the bladder dome and have a reniform shape with an echogenic central interface arising from the collecting system. Loculated fluid collection can mimic the sonographic features of some pelvic masses; however, their irregular shape and unusual location can be depicted.

\section{UTERINE FIBROID}

Uterine fibroids are referred to as fibroid, leiomyoma, leiomyomata, and fibromyoma. They are benign (noncancerous) tumors that grow within the muscle tissue of the uterus. Depending on the prevailing type of tissues- parenchyma or interstitial, they are called differently: myoma, fibroma, fibromyoma, and as they are developed mainly from muscle cells, the most correct terminology is considered to be myoma (leiomyoma) (Figs 9A and B).

Although fibroid (uterine fibroids) is generally considered to be a slowly growing tumor, in $20-40 \%$ of women at the age of 35 and more have uterine fibroids of significant sizes with severe clinical symptoms. Moreover, fibroid can be relapsed in 7-28\% of patients after surgical treatments and in certain cases it may even turn into malignant tumor.

Uterine fibroid is developed on the background of hyper estrogens, progesterone deficits and hyper gonadotropins. The majority of the researchers consider, that the growth of fibroid depends on concentration of cytosolic receptors to the sexual hormones, and their interactions with the endogen or exogenous hormones. In accordance to clinical observations, it can be admitted that both growth and regression of fibroid are estrogen-dependant; the tumor size gets increased during pregnancy and is regressed after menopause.

Fibroids rarely have malignant potential (leiomyosarcoma) (Figs 10A and B).

Fibroid may be located in the external, middle or inner layers of uterus (subserous, interstitial and submucous). Nodules can be located in the isthmus (5\%) or in the uterine body (95\%).

\section{Types of Fibroids}

- Intramural: Fibroids embedded within the myometrium. The most common type of fibroid. These develop within the uterine wall and expand making the uterus feel larger than normal (which may cause "bulk symptoms").

- Subserosal: Fibroids that bulge on the outside. These fibroids develop in the outer portion of the uterus and continue to grow outward. 

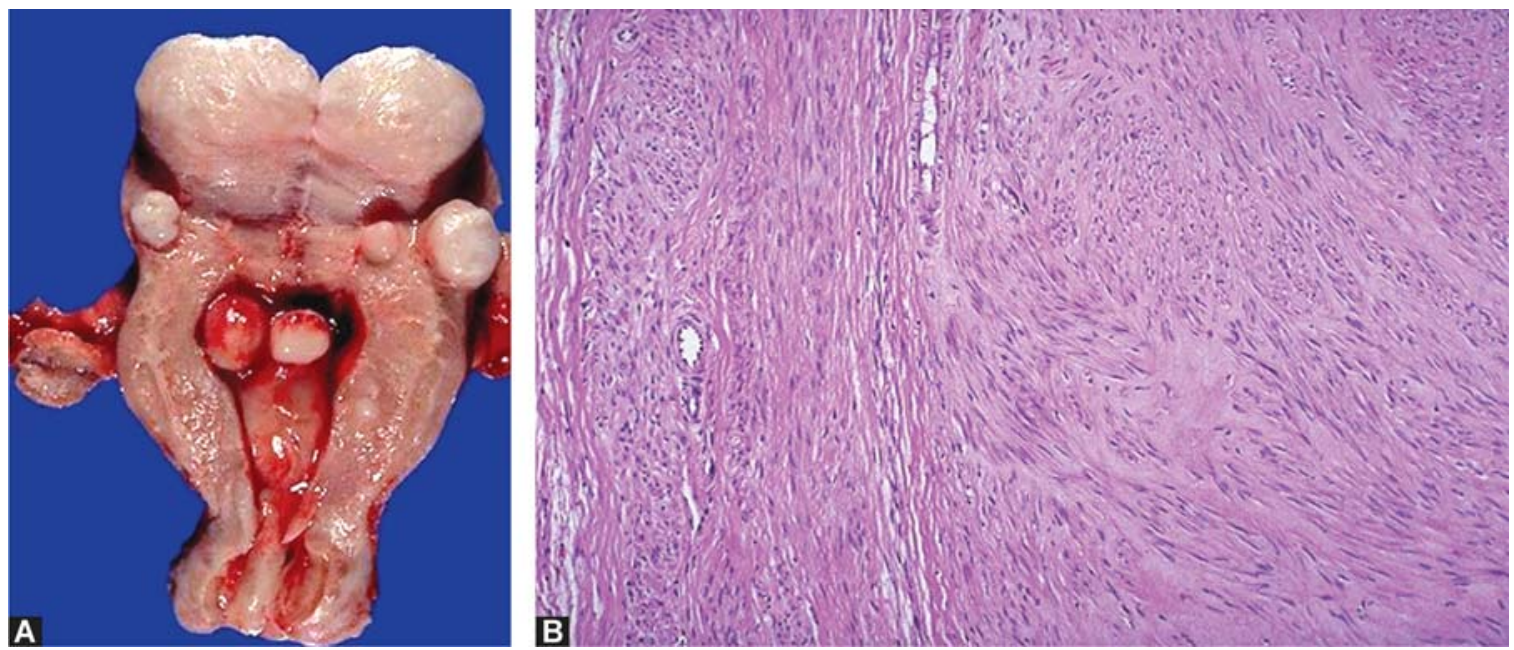

Figs 9A and B: Various types of fibroid and histopathological picture. Proliferation of muscle tissue surrounded by pseudocapsule can be seen. Fibroids rarely have malignant potential (leiomyosarcoma)
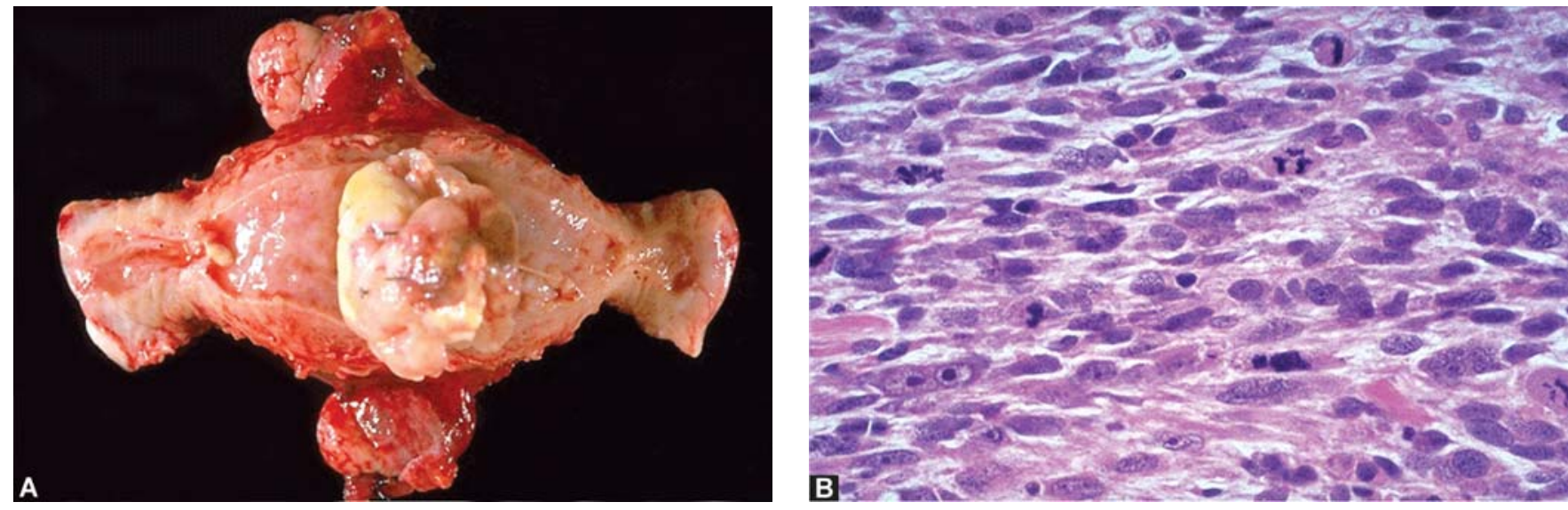

Figs $10 \mathrm{~A}$ and $\mathrm{B}$ : Leiomyosarcoma with histopathological picture

- Submucosal: Fibroids that grow into the inner cavity. These fibroids develop just under the lining of the uterine cavity. These are the fibroids that have the most effect on heavy menstrual bleeding and the ones that can cause problems with infertility and miscarriage.

- Pedunculated: Fibroids that hang from a stalk inside or outside of the uterus. Fibroids that grow on a small stalk that connects them to the inner or outer wall of the uterus (Fig. 11).

Women with fibroids can be asymptomatic (in 50 to $60 \%$ of cases) or may present with menorrhagia (30\%), pelvic pain with or without dysmenorrhea or pressure symptoms (34\%), infertility (27\%), and recurrent pregnancy loss (3\%). Much of the data describing the relationship between the presence of fibroids and symptoms are based on uncontrolled studies that have assessed the effect of myomectomy on the presenting

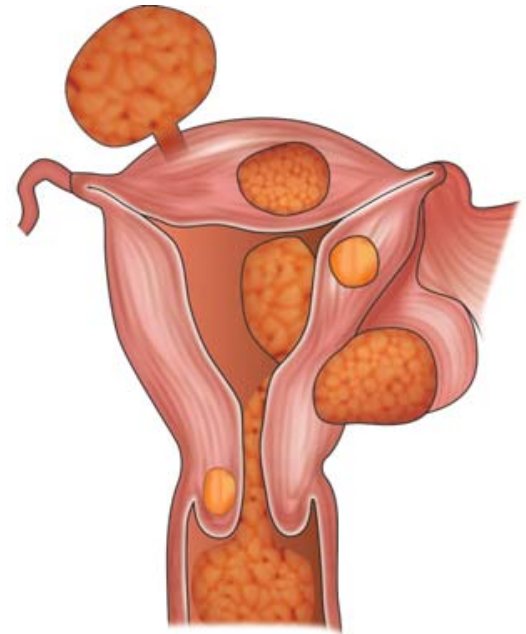

Fig. 11: The names of fibroids reflect their orientation to the uterine wall 


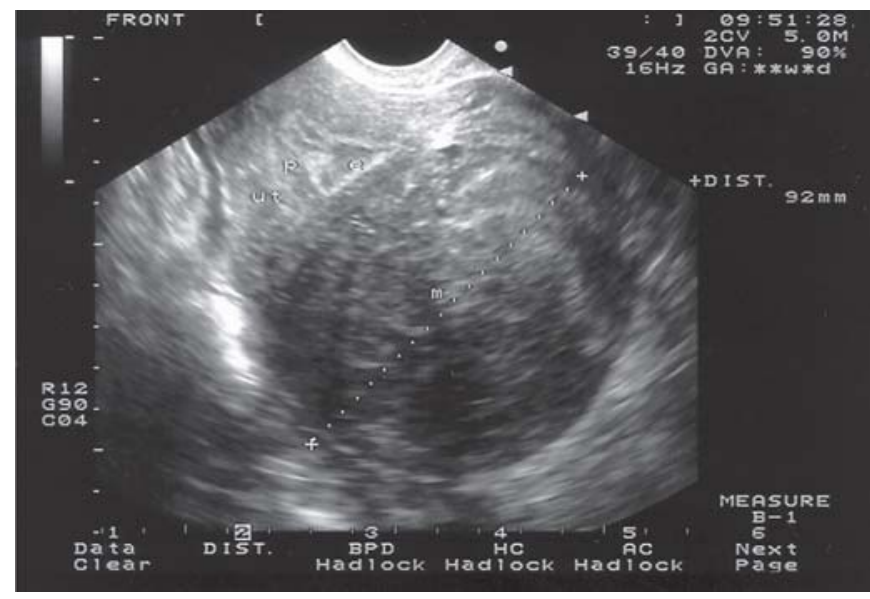

Fig. 12: 2D ultrasound of intramural fibroid. Possible predictor of the uterine growth is fibroid circulation, which can be assessed by color Doppler examination
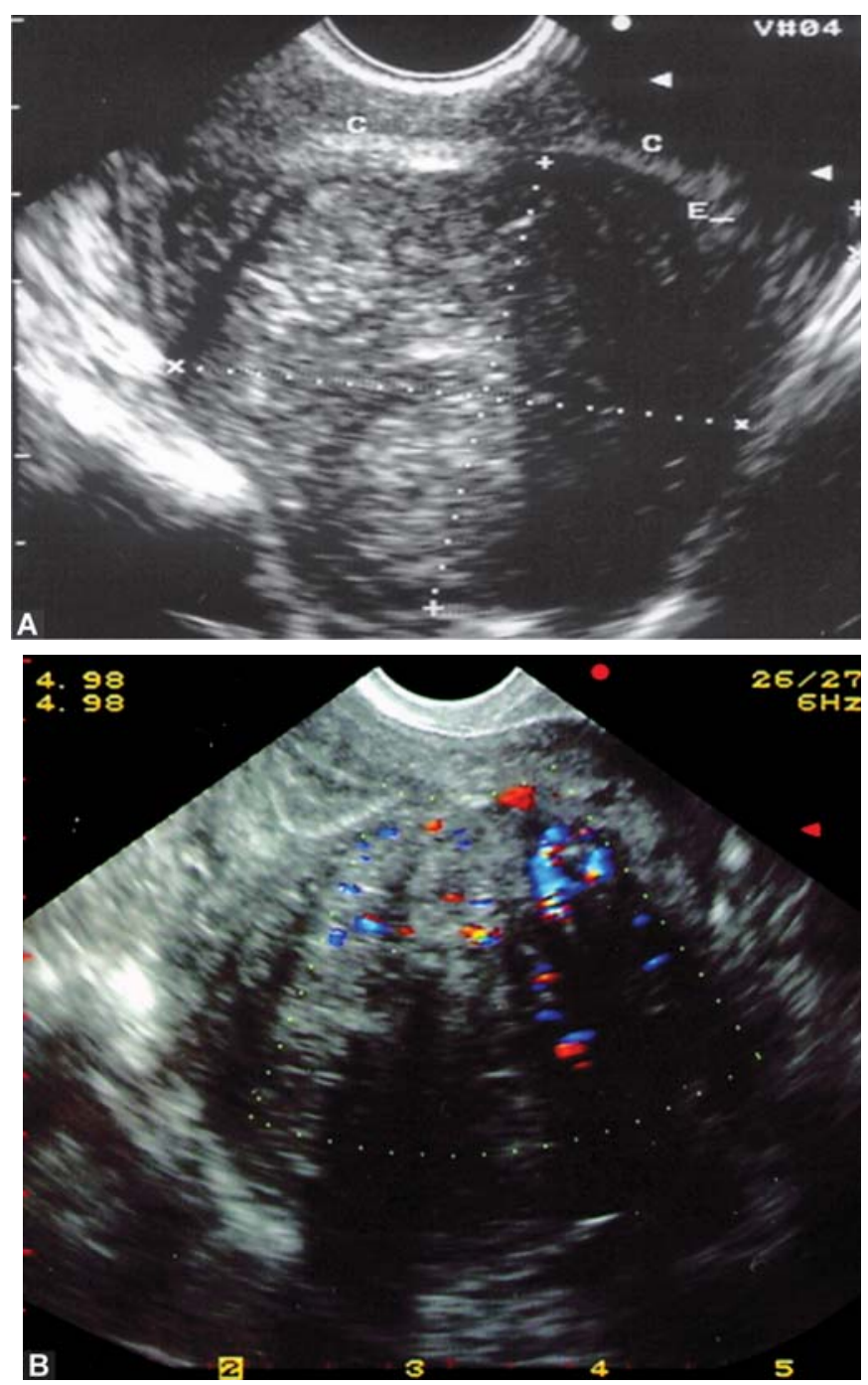

Figs $13 \mathrm{~A}$ and $\mathrm{B}$ : Examination of the fibroid circulation symptoms. The prevalence of fibroids in infertile women can be as high as 13 percent, but no direct causal relationship between fibroids and infertility has been established.

Main fibroid symptoms bleeding, pressure, pain as well as infertility and pregnancy complications, such as cesarean delivery, breech presentation, malposition, preterm delivery, placenta previa, and severe postpartum hemorrhage occur in half of the patients.

Symptoms depend on location, size, growth rate and relation with surrounding organs. Ultrasound is of the most importante diagnostic tool in determining all the previous facts.

While making an ultrasound diagnosis, it is important to determine size, shape echogenicity and clear edge with the surrounding tissues. For that purposes we can use 2D, 3 and 4D. In order to asses the vascularization of the fibroids, color Doppler is used (Figs 12 to 13B).

Endometrial and subendometrial blood flow measured by three-dimensional power Doppler ultrasound in patients with small intramural uterine fibroids during IVF treatment, can be a predictor of a treatment success.

The circulation can be even better assessed using new 3D technologies such as glass body rendering (Figs 14A to 15C).

Using of 3 and 4D ultrasound enables us better delineation of the fibroid. ${ }^{5,6}$

Submucosal fibroids grow into the inner cavity. Ultrasound is of great value in assessing the operability of submucosal fibroids. Contrast ultrasound is of greater efficacy in better diagnosis of submucosal fibroids (Figs 16A to 17C).

In differential diagnosis it is most important not to miss diseases, such as gestational trophoblastic neoplasm or endometrial carcinoma (Figs 18 and 19).

\section{MAGNETIC RESONANCE GUIDED FOCUSED ULTRASOUND}

Magnetic resonance guided focused ultrasound (MRGFU) is a noninvasive outpatient, procedure that uses high intensity focused ultrasound waves to ablate (destroy) the fibroid tissue. During the procedure, an interventional radiologist uses magnetic resonance imaging (MRI) to see inside the body to deliver the treatment directly to the fibroid. The procedure is FDA approved for treating uterine fibroids. MRI scans identify the tissue in the body to treat and are used to plan each patient's procedure. MRI's provide a three-dimensional view of the targeted tissue, allowing for precise focusing and delivery of the ultrasound energy. MRI also enables the physician to monitor tissue temperature in real-time to ensure adequate but safe heating of the target. Immediate imaging of the treated area following MRGFU helps the physician determine if the treatment was successful. ${ }^{11}$ 

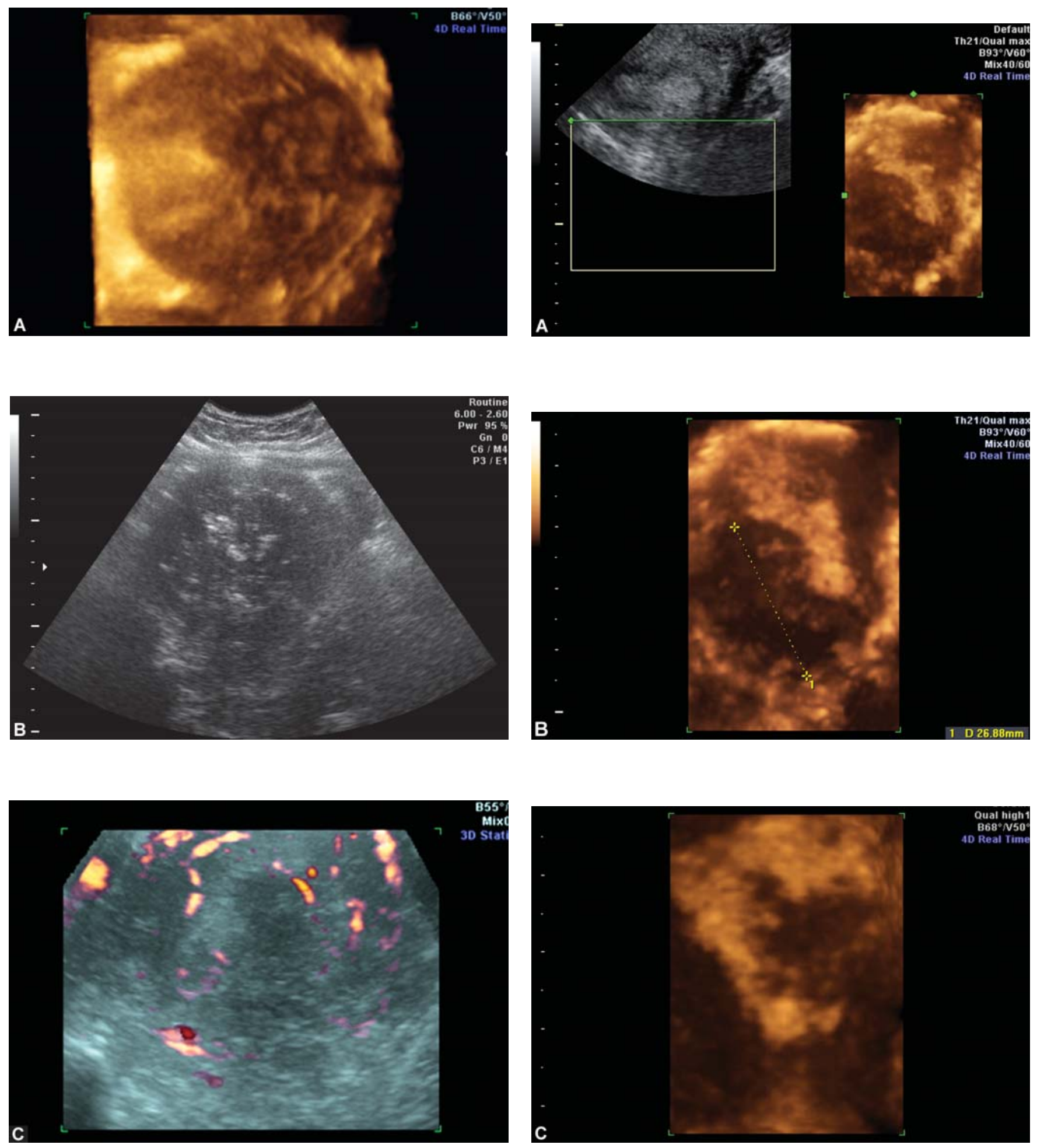

Figs 14A to C: Three-dimensional and glass body picture of the uterine fibroid

Figs 15A to C: Three-dimensional ultrasound picture of the uterine fibroid 

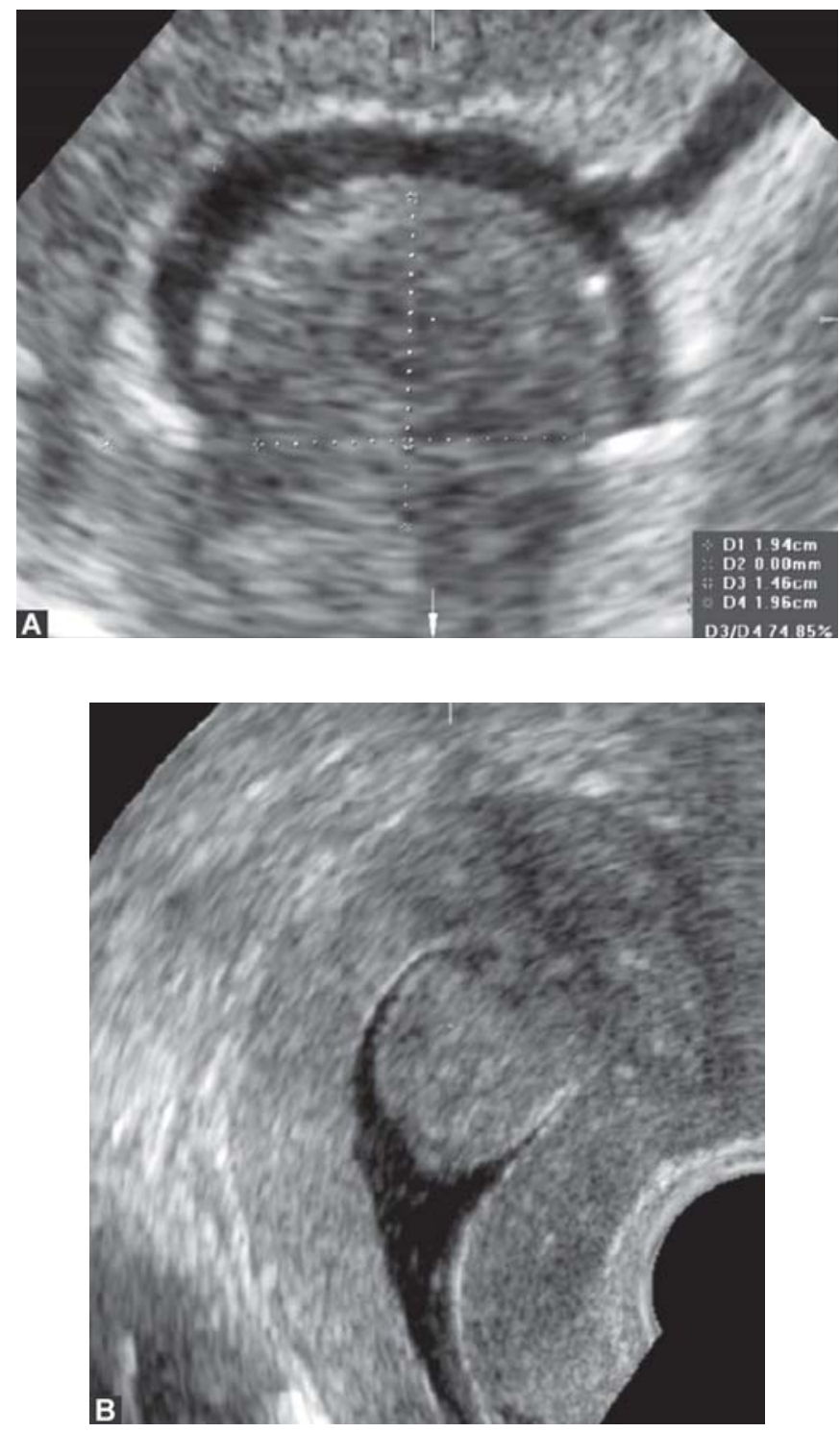

Figs 16A and B: Contrast sonography in diagnosis of submucosal fibroids

The ultrasound energy used in MRGFU can pass through skin, muscle, fat and other soft tissues. High-intensity ultrasound energy that is directed to the fibroid heats up the tissue and destroys it. This method of tissue destruction is called thermal ablation (Figs 20A and B).

\section{ULTRASOUND GUIDED FIBROID SCLEROSATION}

Several years ago, authors presented a new method of myoma therapy: sonographically guided vascular sclerosation. Sclerosation was performed under color doppler sonographic visualization, after circulation mapping. Sclerosation was
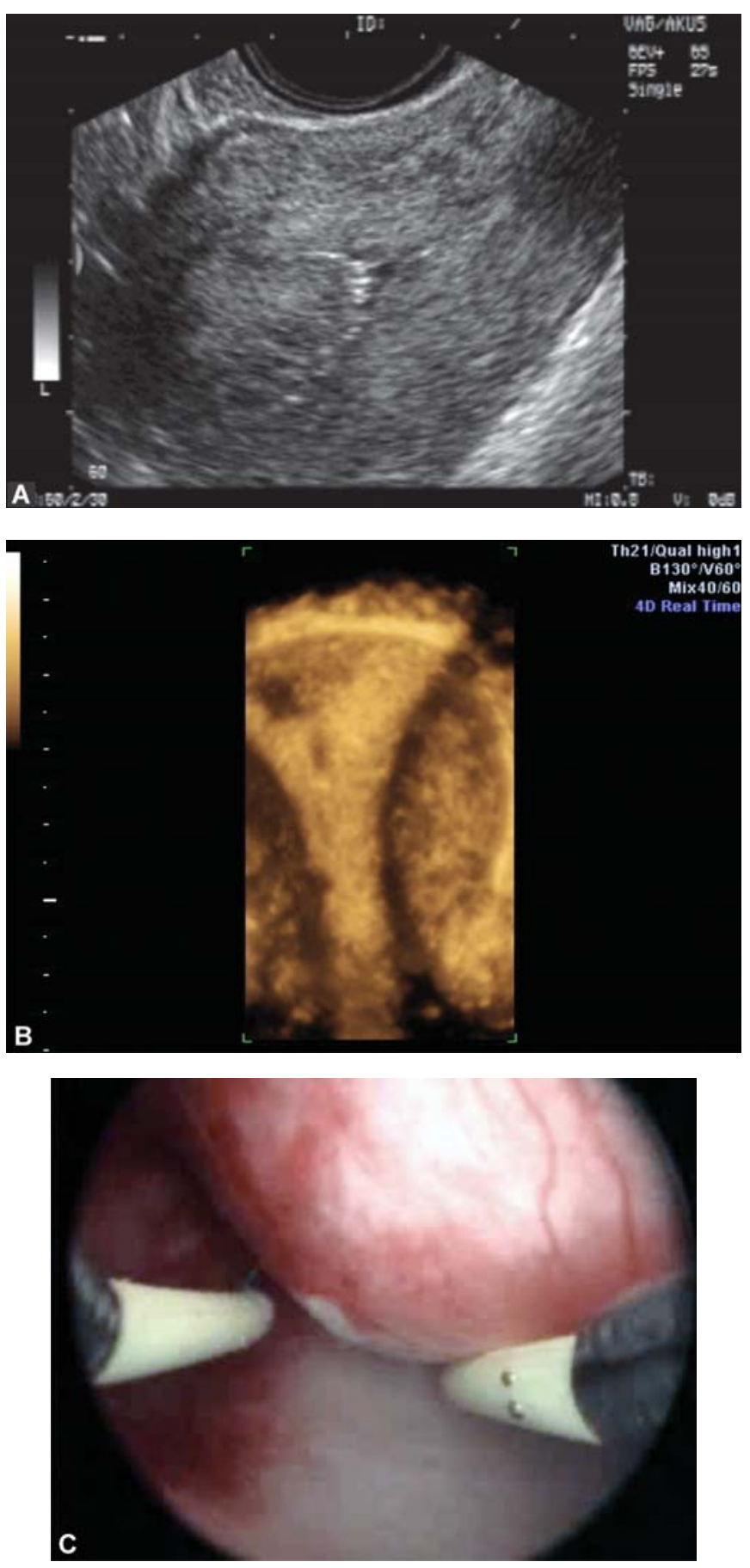

Figs 17A to C: Contrast 2D, 3D and hysteroscopic view of submucosal fibroid

performed with $96 \%$ alcohol and aethynilsclerol (5 to $15 \mathrm{ml}$, depending of the fibroid volume). There was no difference in age, size, location, vascularization and degeneration of uterine fibroids. Indication for treatment (bleeding, pain, pressure) 

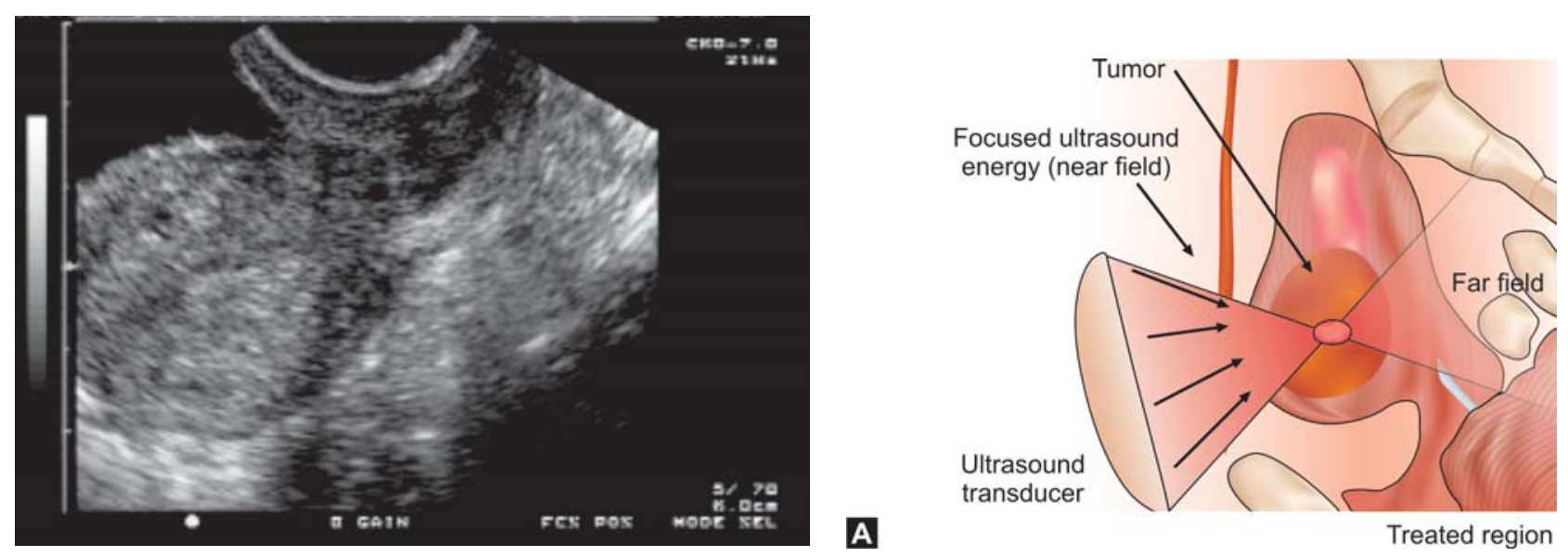

Fig. 18: Endometrial cancer

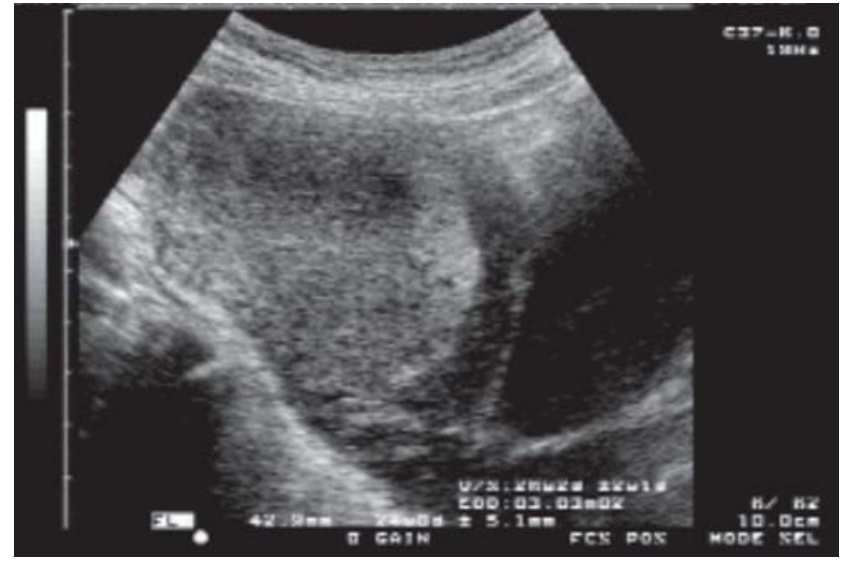

Fig. 19: Gestational trophoblastic neoplasm

showed no difference. Sclerosation was intravascular in 15\% and perivascular in $85 \%$ of patients. During procedure, pain was present in $20 \%$, burning in $85 \%$ and bleeding in $15 \%$.

Procedure had to be repeated in $30 \%$, and it was done three times in $15 \%$ of patients. There was decrease in vascularization (in $85 \%$ ), and in size (in 95\% of patients) after vascular sclerosation. After sclerosation, decrease in bleeding occurred in $75 \%$ of patients, $65 \%$ had less pain, and $80 \%$ decreased pelvic pressure. Hospitalization was significantly shorter 1,5 days and 6,3 days, as well as ICU treatment 0 and 1,5 days, and time to return to work 3 days and 33 days, in groups A and B, respectively. Authors have concluded that sonographically guided vascular sclerosation was safe and effective myoma treatment. It has less complications and shorter recovery time (Figs 21A and B).

Ultrasound is of great use in making a diagnosis of the uterine fibroids, assessing the circulation, making a treatment choice and monitor the therapy effects.

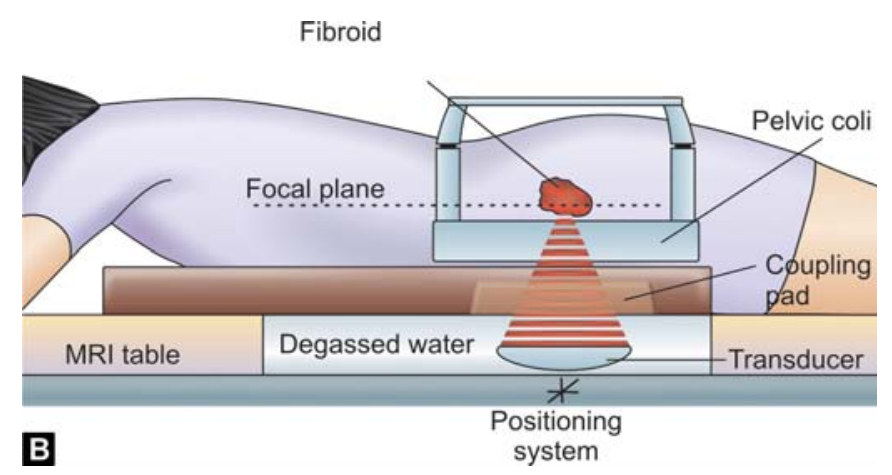

Figs 20A and B: Focused ultrasound surgery-schematic representation

\section{SUMMARY}

This chapter has discussed and illustrated pertinent points in the differential diagnosis of pelvic masses by sonography. Although the sonographic features of a pelvic mass may not allow a specific diagnosis, clinically useful information can usually be obtained. In general. Transvaginal approach is a useful adjunct to transabdominal because it adds specificity in determining intraversus extraovarian masses and endometrial and myometrial disorders. Transvaginal sonography affords an accurate means for evaluation of the ovaries and is particularly useful in obese, postmenopausal women in whom the incidence of ovarian carcinoma is especially high. Color Doppler sonography seems to be helpful in distinguishing benign from malignant ovarian masses and in the evaluation of adnexal torsion. It is particularly useful as an adjunct to morphologic assessment of ovarian lesions. 

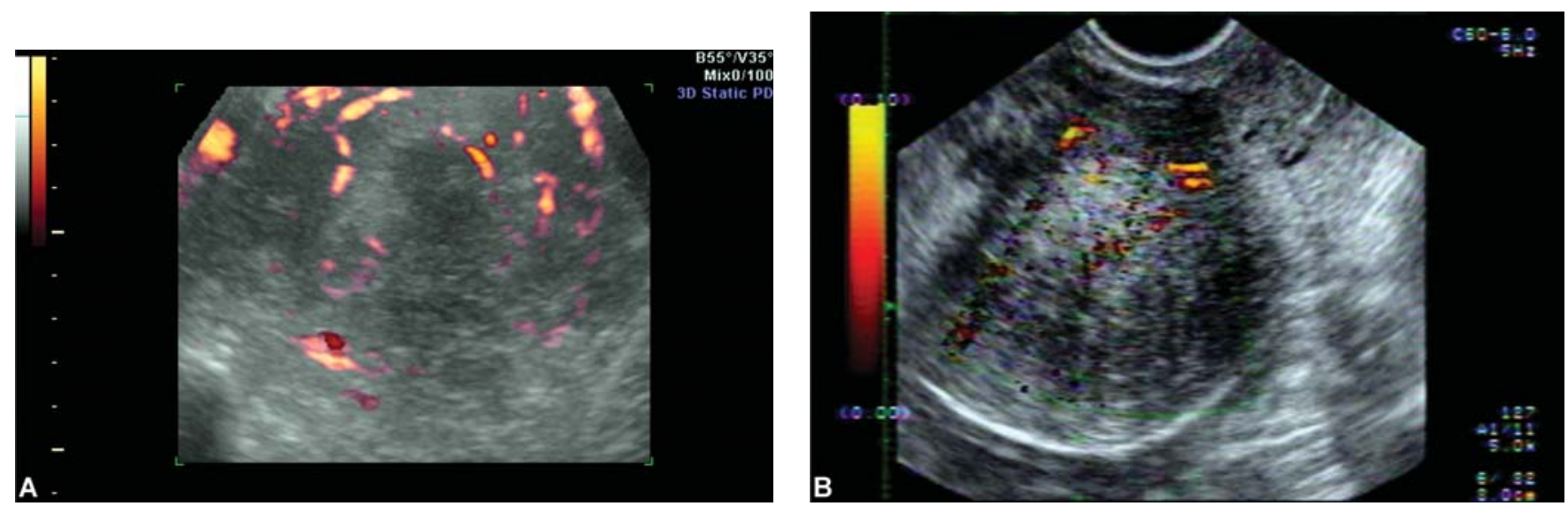

Figs 21A and B: Glass body picture of the uterine fibroid after ultrasound guided fibroid sclerosation

\section{REFERENCES}

1. Imaging in gynecology. Valentin L. Best Pract Res Clin Obstet Gynaecol Dec 2006;20(6):881-906. Epub 2006 Aug 10. Review.

2. Milicevic S, Vilendecic Z, Dokic M, Radunovic N, Stamenovic S, et al. Heterotopic pregnancy: Still a diagnostic puzzle. J Obstet Gynaecol May 2008;28(4):458-59.

3. Medeiros LR, Rosa DD, da Rosa MI, Bozzetti MC. Int J Gynecol Cancer Feb 2009;19(2):230-36.
4. Kurjak A, Jurkovic D, Alfirevic Z, Zalud I. Transvaginal color Doppler imaging. J Clin Ultrasound May 1990;18(4):227-34.

5. Coyne L, Jayaprakasan K, Raine-Fenning N. 3D ultrasound in gynecology and reproductive medicine. Womens Health (Lond Engl) Sep 2008;4(5):501-16.

6. Kurjak A, Kupesic̀ S. Three dimensional ultrasound and power Doppler in assessment of uterine and ovarian angiogenesis: A prospective study. Croat Med J Sep 1999;40(3):413-20. 